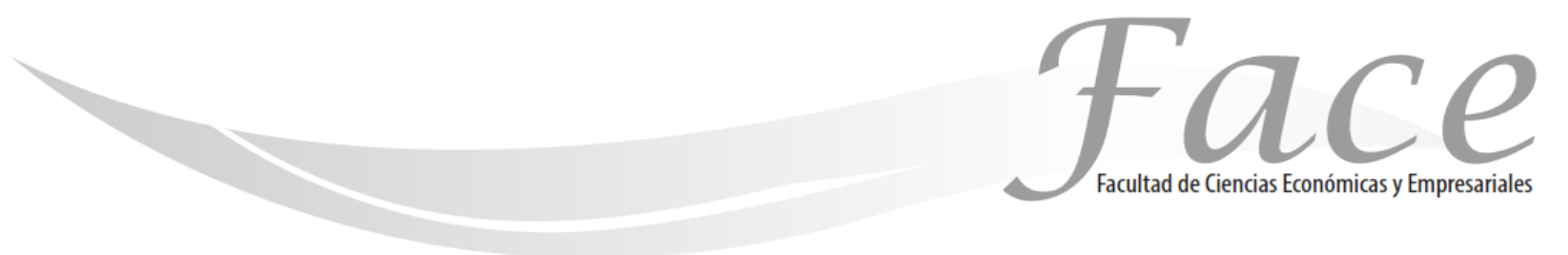

ISSN Impreso: 1794-9920

ISSN Electrónico: 2500-9338

Volumen $16-\mathrm{N}^{\circ} 1$

Año 2016

Págs. $40-47$

\title{
RESPONSABILIDAD SOCIAL EN LAS UNIVERSIDADES PÚBLICAS BINACIONALES DE COLOMBIA Y VENEZUELA
}

\author{
Jorge Fidel Navarro Becerra* \\ Enlace ORCID: http://orcid.org/0000-0001-9610-3351 \\ Yamelys Navarro Becerra** \\ Enlace ORCID: http://orcid.org/ 0000-0002-2831-1738
}

Fecha de Recepción: 31 de Marzo Octubre 2016

Fecha de Aprobación: 10 de Junio 2016

\begin{abstract}
Resumen:
En la presente investigación, se buscó analizar la responsabilidad social en el personal administrativo de las Universidades públicas binacionales. Metodológicamente, fue de tipo descriptiva - cuantitativo; con un diseño no experimental, transeccional descriptivo - de campo. La población fue determinada por dos universidades: Universidad Popular del Cesar- Colombia y la Universidad del Zulia (LUZ), - Venezuela, teniendo unidades informantes de 97 sujetos entre directivos y personal administrativo que trabajan dentro de estos departamentos en los recintos universitarios mencionados. El instrumento de recolección que se empleó fue un cuestionario compuesto por 48 ítems planteados bajo la escala de Likert, con cinco opciones de respuesta y una ponderación de 5 a 1 . Para la validación de contenido se procedió a consultar a 5 expertos. El cual demostró ser confiable según el coeficiente de Cronbach $a=0,92$. El resultado que se obtuvo en términos generales fue la importancia de la responsabilidad social enmarcada en los elementos humanos y ambientales, regida además por una serie de regulaciones legales que garanticen su práctica dando respuestas con políticas de salud, de formas de trabajo, de recursos de adiestramientos, ética, valores morales, fundamentos de la institución, planes activos basados en el bienestar social y en el desarrollo sustentable de las sociedades. Por ello se recomendó, darle un mayor seguimiento a todas estas acciones de la responsabilidad social y a su fiel cumplimiento.
\end{abstract}

Palabras Claves: Responsabilidad Social, Sociedad, Medio Ambiente, Universidad, partes interesadas.

\footnotetext{
*Profesional en Administración de empresas, especialista en Gerencia de mercadeo, de la Universidad Jorge Tadeo Lozano, Bogotá Colombia Magister en Gerencia de recursos humanos de la Universidad privada Dr. Rafael Belloso Chacín. Contacto: Jfnb1229@gmail.com

** Profesional en Ingeniería Agroindustrial, Magister en Ciencias Ambientales, Candidata a Doctora en Ciencias gerenciales de la Universidad privada Dr. Rafael Belloso Chacín. Contacto: Ynavarro08@infotep.edu.co
} 


\title{
SOCIAL RESPONSIBILITY IN PUBLIC UNIVERSITIES AND VENEZUELA COLOMBIA BINATIONAL
}

\begin{abstract}
:
In this research, we sought to analyze the social responsibility in the administrative staff of binational public universities. Methodologically, it was descriptive - quantitative; with a non-experimental, transeccional design - descriptive - field. The population was determined by two universities: Popular University of Cesar Colombia and the University of Zulia (LUZ) Venezuela, with 97 subjects reporting units between managers and staff working within these departments in the aforementioned campuses. The collection instrument that was used was a questionnaire composed of 48 items raised under the Likert scale, with five response options and a weight of 5 to 1 . For content validation proceeded to consult experts 5 . Which he proved to be reliable according to the Cronbach $a=0.92$. The result obtained in general terms was the importance of social responsibility framed in human and environmental elements, also governed by a series of legal regulations that guarantee their practice giving answers with health policies, ways of work, resources trainings, ethics, moral values, foundations of the institution, active plans based on social welfare and sustainable development of societies. It was therefore recommended greater monitoring to give all these actions of social responsibility and its faithful implementation.
\end{abstract}

Keywords: Social Responsibility, Society, Environment, University stakeholders.

\section{RESPONSABILIDADE SOCIAL EM UNIVERSIDADES PÚBLICAS E VENEZUELA COLOMBIA BINACIONAL}

\section{Resumo:}

Esta publicação teve como objetivo analisar a responsabilidade social no pessoal administrativo das universidades públicas binacionais. Metodologicamente, foi descritiva - quantitativa; com um não-experimental, design transeccional - descritivo campo. A população foi determinada por duas universidades: Universidade Popular de Cesar - Colombia e da Universidade de Zulia (LUZ) - Venezuela, com 97 indivíduos unidades entre gerentes e funcionários que trabalham dentro desses departamentos nos campi acima referidos relatórios. 0 instrumento de coleta utilizado foi um questionário composto de 48 itens captados no âmbito da escala de Likert, com cinco opções de resposta e uma ponderação de 5 para 1. Para validação de conteúdo, procedeu a consultar os peritos 5. Que ele provou ser confiável, de acordo com a a Cronbach $=0,92$. 0 resultado obtido em termos gerais, foi a importância da responsabilidade social enquadrado em elementos humanos e ambientais, também governada por uma série de normas legais que garantam a sua prática dando respostas com as políticas de saúde, formas de trabalho, recursos treinamentos, ética, valores morais, fundações da instituição, planos ativos baseados em bem-estar social e desenvolvimento sustentável das sociedades. portanto, foi recomendado um maior acompanhamento a dar todas estas ações de responsabilidade social e sua implementação fiel.

Você deve criar um departamento exclusivo de responsabilidade social anexado ao Vice-Reitor de Extensão Universitária, onde eles tomam em conta os aspectos internos e externos, e, assim, garantir o pleno cumprimento da responsabilidade social.

Palavras-Chave: Responsabilidade Social, Sociedade, Meio Ambiente, Universidade, partes interessadas. 


\section{INTRODUCCIÓN:}

En la actualidad las organizaciones deben adaptarse a las exigencias de la sociedad, por cuanto se dinamiza en los avances que suceden en el ámbito organizacional 0 empresarial. De esta realidad no escapan las instituciones de educación superior, donde se interrelacionan los factores de producción intelectual, para establecerse una coordinación efectiva entre sus integrantes y la ejecución de tareas, cuyo objetivo final es el logro del desarrollo integral del hombre, para transformarlo en un individuo crítico, reflexivo, autónomo capaz de propiciar el crecimiento económico, cultural, tecnológico y social del país. Existen muchos casos, donde los gerentes de las Universidades, bien sea públicas o privadas, adoptan la responsabilidad social como una obligación, considerando a su vez, que su principal objetivo es dirigir la compañía en búsqueda de conseguir los beneficios de sus accionistas, frente a su contribución con la sociedad, se limita al pago de impuestos al gobierno, para determina legalmente el aprovechamiento, igualmente la utilización de los recursos disponibles en esta, siempre que puedan dedicarse a la competencia libre y abierta. Sin embargo, la sociedad actual sostiene que las Universidades, por ser entes sociales importantes e influyentes, les incumbe la responsabilidad de tanto mantener y mejorar el bienestar global de la sociedad en general, buscando así conocer, contribuir y ayudar socialmente a la comunidad en general; siendo esto obvio, según expresiones del autor Rivera (2006), quien señala que la Responsabilidad Social, conduce a llevar a cabo actividades dirigidas a ayudar a la sociedad, aun cuando estas actividades no contribuyan directamente a generar beneficios directos para las mismas. En otras palabras, tal en lo esbozado en el Libro Verde de las Comunidades Europeas (2010), donde se señalan eventos 0 actividades en las que se involucra interna o externamente la empresa, en la búsqueda del desarrollo social sostenible, ambiente y seres vivos, a objeto de asegurar las necesidades del presente sin comprometer la capacidad de las futuras generaciones para enfrentarse a sus propias necesidades, propiciando la posibilidad de compatibilizar el crecimiento económico con la preservación ambiental; en otras palabras, producir más, consumir menos recursos y generar menos residuos, la vía alterna para mejorar las condiciones de vida en general. Por ende, actualmente, las empresas sea cual sea su ramo o actividad económica, han adoptado el término de responsabilidad social, como presión del ámbito mundial y efectos de la globalización. De allí, que este fenómeno exige de dichas empresas una responsabilidad social; es decir, que la sociedad no las vea como entes con afán de lucro, sino entes sociales generadoras de mejor calidad de vida, protectoras del medio ambiente, comprometidas con las mejoras de los servicios, desarrollo de programas compensadores en las acciones, las cuales, como producto de sus actividades o procesos productivos, pudiesen haber afectado el ambiente y la sociedad en general. Bajo estas circunstancias, cuando las sociedades reclaman una mayor responsabilidad social de sus instituciones, es imperativo que las universidades desplieguen un papel protagónico, asumiendo la información y el conocimiento de un recurso económico, tanto el generado internamente por el talento y la creatividad de su capital humano, el derivado de las políticas encaminadas a cumplir con la investigación y la misión de extensión, lo cual debe ser objeto de una acertada gestión, debido a la incidencia directa con el desarrollo del ámbito sociopolítico para un contexto local, cada vez más global. De esta realidad, no escapan las Universidades binacionales, quienes durante los últimos años han introducido la responsabilidad social como respuesta estratégica asumidas por las mismas, para así sintonizarse con las exigencias de la realidad contemporánea que guardan relación con las ideas de retribuir, compartir, contribuir y corresponsabilizar a las organizaciones con el objeto de devolverles de alguna manera las condiciones así como posibilidades que la sociedad les ha aportado. Por ende, el desarrollo socioeconómico de un país está estrechamente relacionado con el sistema educativo, por buscar soluciones a los problemas y necesidades más apremiantes de la sociedad. Por lo tanto, en Colombia y Venezuela, se presentan y se agudizan cada vez más la violencia, la desigualdad social, la pobreza, temas de interés o controversia entre los muchos estamentos, gobierno, empresa, instituciones, universidades, buscando afanadamente dar respuesta oportuna y eficaz a esta problemática social. Por ello, la conveniencia de sistematizar la responsabilidad social de las Universidades, como estrategia de cambio en la solución novedosa de los problemas sociales, mediante la identificación de nuevos servicios, planes o programas para mejorar la calidad de vida de las comunidades; también la identificación de nuevos formas de participación, organización y movilización como elementos que contribuyan a mejorar la posición social de los pueblos.

\section{MARCO TEÓRICO:}

La Responsabilidad Social, en sus comienzos, se inicia con una perspectiva solamente empresarial, sin embargo hoy en día se plantea en todos los ámbitos de la sociedad. Es una práctica donde los actores sociales asumen su compromiso de responder a sus actividades, respondiendo a los ámbitos sociales, económicos y ambientales del entorno, garantizando la generación equitativa, sostenible y sustentable de beneficios para las partes interesadas. Asimismo, durante los últimos años el tema de la responsabilidad social, según lo mostró Torres (2014), quien en su publicación, manifiesta haber tomado una 
creciente importancia a nivel internacional, pero al mismo tiempo ha generado un fuerte debate sobre sus alcances e implicaciones, sobre todo por la aparente dificultad de poder llevar a la práctica un concepto que incide de manera directa en la imagen de la empresa en la sociedad, poniendo en juego su reputación y, con ella, la confianza y el respaldo de los diferentes grupos de interés que la componen. Todo ello dentro de un entorno de negocios globalizado en el que se han incrementado las demandas sociales sobre el papel desempeñado por las empresas, el impacto de sus actividades en la sociedad y en el medio ambiente. Este nuevo enfoque en la manera de hacer negocios ha hecho replantear los esquemas tradicionales de gestión empresarial, propiciando la gerencia del cambio organizacional basada en los planteamientos de la responsabilidad social, haciéndolos coincidir con el objetivo fundamental de convertir la empresa en un motor de desarrollo económico, pero de manera ética y socialmente responsable, sin priorizar el beneficio 0 la utilidad económica de la inversión sobre aspectos reprochables desde un punto de vista social. Así la responsabilidad social es la contribución activa y voluntaria de las empresas al mejoramiento social, económico y ambiental. Bajo este concepto de administración se engloban un conjunto de prácticas, estrategias y sistemas de gestión empresariales que persiguen un nuevo equilibrio entre las dimensiones económica, social y ambiental aplicables a todo tipo de organización, incluidas las educativas. La responsabilidad social según García (2006), quien la describe como "la obligación gerencial de tomar las medidas para proteger y mejorar el bienestar de la sociedad como un todo y también los intereses organizacionales. Se deduce, que este término hace referencia al conjunto de obligaciones y compromisos legales y éticos del personal directivo, nacionales y regionales derivados de los impactos de las actividades de las escuelas, las cuales se producen en el ámbito social, laboral, medioambiental y de los derechos humanos. De acuerdo con la definición de la Comisión Europea en su Libro Verde: Fomentar un marco europeo para la responsabilidad social de las empresas (2010, p. 45), donde se señala que la Responsabilidad Social "[...] es la integración voluntaria, por parte de las empresas, de las preocupaciones sociales y medioambientales en sus operaciones comerciales y sus relaciones con sus interlocutores, igualmente el autor antes mencionado plantea que ser socialmente responsable no significa cumplir plenamente las obligaciones jurídicas, sino también ir más allá de su cumplimiento invirtiendo "más" en el capital humano, el entorno y las relaciones con sus interlocutores. De allí, que el imperativo de la responsabilidad social es particular para cada localidad, por ello depende del contexto de acción. En los países desarrollados las prioridades son: la protección al medio ambiente, atención al público interno, así como generar en los consumidores una conciencia social, mientras en las naciones con economías emergentes la pobreza es el principal problema a resolver. Ahora bien, en cuanto a la Responsabilidad Social dentro de las Universidades, han escrito varios autores entre ellos se ha destacado el escritor peruano Francois Vallaeys (2007), quien ha fortalecido la conceptualización en este campo, a través de docentes que se preparan de forma virtual en cursos sobre ética, capital social y desarrollo en la Universidad. En este sentido, Nieto y Fernández (2004), citados por Guerra (2007), quienes expresan que existen dos corrientes de pensamiento de la Responsabilidad Social una completamente financiera, donde los objetivos socioeconómicos son incompatibles, teniendo en Milton Friedman uno de los primeros propulsores, quien acentúa su atención en los accionistas (shareholders), considerando que los directivos deben actuar como agentes, defendiendo los intereses de los propietarios de la empresa. Por otra parte, los citados autores hablan sobre otra corriente teórica contraria a la Friedman, la de Kenneth Arrow, quien afirma que cuando no se restringe la maximización como objetivo básico empresarial, la distribución de los beneficios es bastante inequitativa, siendo lo deseable manejar un concepto de responsabilidad social por parte de las empresas, donde estas experimenten una obligación ética, moral o legal. Analizados los dos enfoque anteriores, se puede argumentar que el comportamiento socialmente responsable, no es incompatible con la creación de valor y con la filosofía organizacional de responsabilidad social, para tomar en cuenta los intereses de todos los participantes (stakeholders) en el sistema empresarial. Desde esta perspectiva, se infiere a las gerencias de las organizaciones la búsqueda de un equilibrio en los intereses de todos los grupos tanto de la dimensión interna y como de la externa a la empresa. En este orden de ideas, se puede precisar que la responsabilidad social va más allá de obligación de la empresa porque, debe ser asumida con un compromiso, participación y colaboración consciente deliberadamente hacia un colectivo que enfrenta serios problemas económicos, sociales ambientales. De lo expuesto en los párrafos anteriores y analizando los problemas sociales de la actualidad, se evidencia el fracaso del modelo tradicional, que defendía Friedman por lo cual se considera conveniente cambiar el enfoque por el de una filosofía empresarial de responsabilidad social, que tome en cuenta los intereses de todos los participantes en el sistema organizacional, con la identificación y ejecución de principios básicos y complementarios, acordes con los fines sociales, incluidos en la estrategia y acciones frecuentes. Es importante acotar, que además de este comportamiento, se debe percibir en los empleados, accionistas, empresarios y trabajadores una conducta ética en todos los ámbitos de acción de la organización. 


\section{METODOLOGÍA:}

Según la naturaleza de los objetivos a alcanzar, fue considerado como un estudio tipo descriptivo - cuantitativo; con un diseño descriptivo, de campo, no experimental transeccional dado que se buscó analizar las características de la responsabilidad social en el personal administrativo de las Universidades públicas binacionales, sin manipular la variable, en su área directamente, en un tiempo determinado, que fue durante el año 2015, la población estuvo constituida por las Universidades públicas de Valledupar Cesar - Colombia y Maracaibo - Venezuela. Se usó como herramienta discriminante que éstas fuesen recintos universitarios públicos, ubicados en municipios de las principales ciudades, arrojando un número de dos (02), las cuales fueron: la Universidad Popular del Cesar, Vicerrectoría Administrativa- Valledupar Cesar - Colombia y la Universidad del Zulia (LUZ), (sede rectoral) del Municipio Maracaibo - Venezuela. Bajo esas premisas, se tuvo que la población quedó constituida por 97 empleados y directivos conformando esto las unidades de análisis, representando una población finita. Como herramienta de apoyo para recolectar los datos, se utilizó la encuesta como técnica de recolección de información y como instrumento un cuestionario tipo Likert para la variable: Responsabilidad social en el personal administrativo de las Universidades públicas binacionales, con un sistema de cuarenta y ocho (48) preguntas cerradas que incluyen los ítems necesarios para el estudio, estructurados en dos partes que permitieron obtener la información requerida y de manera confidencial. Estando está conformada por cinco (5) opciones de respuesta desde Siempre (S), hasta Nuca $(\mathrm{N})$, con una ponderación de cinco a uno, cuya ponderación máxima alcanzada por un sujeto fue de 255 puntos y la mínima fue de 51 .

\section{RESULTADOS Y DISCUSIÓN:}

Los resultados obtenidos de la aplicación del cuestionario dirigido a los sujetos que trabajan como directores y personal administrativo de la Universidad Popular del Cesar, Vicerrectoría Administrativa Balneario Hurtado Vía a Patillal - Valledupar Cesar - Colombia y la Universidad del Zulia (LUZ), (sede rectoral) del Municipio Maracaibo Venezuela, con la finalidad de analizar la responsabilidad social en el personal administrativo en dichos recintos públicos binacionales.

Igualmente es oportuno señalar que, el análisis de los resultados se efectuó mediante la aplicación de estadística descriptiva, empleando para ello las medidas estadísticas de tendencia central, aplicando el paquete estadístico SPSS en Microsoft Office Windows 7. Cabe mencionar que la información recolectada fue analizada de acuerdo a un baremo para la codificación de los estudios de la variable, basándose en los diversos postulados teóricos.

Tabla No 1

Baremo Categorización de los Valores

\begin{tabular}{|c|c|c|}
\hline $\begin{array}{c}\text { Escala de } \\
\text { Valores }\end{array}$ & Alternativas & Categorías \\
\hline $\begin{array}{c}1.00 \ldots \ldots \ldots . .1 .8 \\
0\end{array}$ & Nunca & Ausente \\
\hline $\begin{array}{c}1.81 \ldots \ldots \ldots .2 .6 \\
0\end{array}$ & Casi nunca & Poco presente \\
\hline $\begin{array}{c}2.61 \ldots \ldots \ldots . .3 .4 \\
0\end{array}$ & Algunas veces & $\begin{array}{c}\text { Medianamente } \\
\text { presente }\end{array}$ \\
\hline $\begin{array}{c}3.41 \ldots \ldots \ldots . .4 .2 \\
0\end{array}$ & Casi siempre & Presente \\
\hline $\begin{array}{c}4.21 \ldots \ldots \ldots .5 .0 \\
0\end{array}$ & Siempre & Muy Presente \\
\hline
\end{tabular}

Luego de la codificación de los datos se procedió a analizar los resultados plasmados, corresponden a la dimensión: Elementos; Sub- dimensión Compromiso, en el cual se evidenció el indicador: Adaptabilidad, presentó mayor frecuencia de respuestas en la opción casi siempre con un valor de $57,7 \%$, es decir, los gerentes y personal administrativo de las universidades analizadas, consideran que planifican las acciones en materia de Responsabilidad Social.

Por otra parte el indicador: Desarrollo personal registró una máxima frecuencia en la opción de respuesta nunca, con una representación del 52,6\% los encuestados expresaron que no diseñan objetivos a desarrollar tomando en cuenta los intereses de todos los afectados, a su vez un $26,8 \%$ de la población consideró casi siempre el personal de las universidades pretenden ser socialmente responsable con la realidad de la sociedad de colaboradores a la que pertenece, mientras un $20,6 \%$ respondió algunas veces, ubicado en la categoría ausente según el baremo diseñado por el investigador.

Por último, para el indicador: Seguridad y salud en el trabajo, se obtuvo en la opción de respuesta algunas veces se obtuvo el $49,5 \%$ de la frecuencia, debido a que los directores y empleados del área administrativa de las universidades binacionales analizadas, consideraron el desarrollo de la práctica de actividades socialmente responsable para traer beneficios al crecimiento de la Universidad. Por otra parte, la opción casi nunca registró un $27,9 \%$, mientras la opción casi siempre con un $21,6 \%$ de la frecuencia, este indicador alcanzó una media aritmética de 3,00 indicando, según el baremo, que está 
medianamente presente en los recintos universitarios antes mencionadas.

Seguidamente, la dimensión: Elementos y la SubDimensión: Adaptabilidad al cambio

Evidencian que para el indicador: Ética, los directivos y personal administrativo de las Universidades públicas Binacionales, manifestaron que algunas veces se adaptan al cambio en un proceso de reestructuración para ser socialmente responsable internamente representado con un $41,2 \%$. Así mismo la opción de respuesta casi siempre alcanzó un $40,2 \%$ de frecuencia relativa, la alternativa siempre un $10,3 \%$ y un $8,2 \%$ respondió casi nunca, mientras la opción de respuesta nunca no estuvo presente. Asimismo, el indicador: Liderazgo, según la población seleccionada de las universidades públicas binacionales analizadas, se observó la mayor frecuencia de respuestas en la alternativa algunas veces con un 50,5\%, lo cual indicó que los gerentes del área se preocupan porque su personal participe en cursos de adaptación al cambio para ser socialmente responsable, un $39,2 \%$ opinaron que casi nunca, un $9,30 \%$ dijo que casi siempre, sólo un 1,00\% seleccionó la opción nunca mientras que para la alternativa siempre no hubo respuestas.

En cuanto a las políticas de Políticas de responsabilidad social, se refiere para el indicador: Participación ciudadana se pudo observar, con una frecuencia de $77,3 \%$ que siempre se considera que en las Universidades públicas analizadas en su proyección social tiene en cuenta a la sociedad. Con una frecuencia de $21,6 \%$ la población manifestó que casi siempre participa la sociedad en los procesos de planificación de la universidad, sólo el 1\% considera que algunas veces, mientras que las alternativas casi nunca y nunca no obtuvieron respuestas. Este indicador obtuvo un promedio de 4,70 lo que evidencia, de acuerdo al baremo seleccionado para esta investigación, en el indicador analizado se encuentra muy presente en las Universidades públicas de Valledupar Cesar - Colombia y Maracaibo Venezuela.

Por otra parte el indicador: Utilización adecuada de recursos, alcanzó la mayor frecuencia de respuestas en la opción siempre con un $85,6 \%$ reflejando como los directores y empleados administrativos de las universidades analizadas a través de una actitud proactiva realizan acciones para que sus miembros permanezcan en la misma. La opción de respuesta casi siempre, obtuvo un $10,3 \%$, algunas veces $4,10 \%$ dejando las alternativas nunca y casi nunca sin respuesta. El promedio para este indicador fue de 4,70 lo que indica está muy presente.

Finalmente, el indicador: Efectos Ambientales, obtuvo un $49,5 \%$ en la opción de respuesta casi nunca, reflejando que la educación ambiental para las universidades públicas binacionales analizadas es fundamental.
Al explicar las características de responsabilidad social que posee el personal administrativo de las Universidades públicas binacionales.

Finalmente en cuanto a la dimensión que tiene que ver con las características de responsabilidad social. El indicador: Atención a problemas de la sociedad, obtuvo la mayor frecuencia de respuesta en las opciones siempre y casi siempre con un valor de $44,3 \%$, lo cual indicó que las universidades analizadas integran su personal a las políticas de recursos naturales a fin de garantizar una responsabilidad social adecuada.

Seguidamente, se pudo observar en la tabla, para el indicador: Promoción de la equidad social, en el cual la mayor frecuencia se ubicó en la opción casi nunca con un valor de $49,5 \%$ que se tradujo en la poca promoción de la tolerancia, el entendimiento mutuo y los valores compartidos en su capital social, es decir, casi nunca se muestran la responsabilidad social por medio de su compromiso con la equidad social.

\section{CONCLUSIONES:}

Al categorizar los constructos teóricos que fundamentaron la responsabilidad social en el personal administrativo en las Universidades públicas Binacionales, a través de los resultados obtenidos de la aplicación del instrumento, se procedió a confrontar la media aritmética de los puntajes correspondientes a las dimensiones; para finalmente, analizar la variable, con la finalidad de equiparar los datos con las bases teóricas que soportaron la investigación, e igualmente se categorizaron según el baremo previamente diseñado, donde se denotó que para la variable analizada se obtuvo un promedio de 3,50, estando presente dentro de las instituciones estudiadas. Por su lado, se pudo indicar que si bien es cierto, los elementos de la Responsabilidad Social, son manejados en un grado aceptable, es necesario aumentarlos y reforzar su promoción definida por comportamientos, aptitudes 0 actitudes responsables-sociales en las diversas situaciones que enfrenten las universidades analizadas. Dichos aspectos permitirán establecer una cultura moral idónea para favorecer y desarrollar el binomio Universidad Comunidad para el crecimiento del país. De allí, que hoy día la demanda de la sociedad en general busca un desarrollo, difusión y aplicación de la responsabilidad social, como requisito indispensable para que una institución sea competitiva en el mercado. Pero no sólo en el mundo empresarial, también en el ámbito organizacional de su recurso humano, este concepto va adquiriendo mayor relevancia de forma paulatina, y en particular, en el panorama universitario, donde el personal de estos recintos universitarios deben ser socialmente responsables 
de los problemas de la sociedad que los rodea. De esa forma, se infiere que las universidades públicas objeto de estudio, aplican instrumentos que conlleven al mejoramiento continuo, así como a la prevención de la contaminación de las comunidades donde operan. Del mismo modo, dirigen las iniciativas hacia sus responsabilidades internas; pero no han logrado integrar las acciones de responsabilidad social, con sus actividades centrales. Es por esas razones enunciadas, que se pudo concluir que la responsabilidad social en las Universidades públicas binacionales, está presente pero no en un nivel óptimo, existe falta de seguimiento, de interés, de aplicación y de propagación para con los trabajadores de las empresas integrantes de dicho sector. Se presentan elementos 0 ámbitos descuidados que forman parte de la estructura de un esquema de desarrollo socialmente responsable y deben ser cubiertos para lograr darle establecimiento a la variable en cuestión. Es un tema que está en la palestra y forma parte de un cambio en la manera de hacer negocios, de contribuir al desarrollo aprovechando al máximo todos los recursos y resguardando el más relevante, el capital humano. De allí, que en relación a lo descrito anteriormente, se enfatiza la necesidad, en la premura de dirigir claramente el apoyo a la formación del recurso humano, dotándolos de asistencia y protección ante circunstancias determinadas. Hacia los trabajadores, los programas sociales que brindan la posibilidad de desarrollarse y de contar con elementos que les aseguren gozar de recursos económicos, de asistencia social, atención a la salud, de mejoramiento personal, teniendo así la oportunidad de concretar una alta calidad de vida garantizando el bienestar de ellos, de sus familiares y de la comunidad en general, porque realmente no se práctica a cabalidad. Cabe destacar que los trabajadores de las instituciones universitarias analizadas, no tienen sentido de pertenencia hacia ellas mismas, ya que se evidenció que no tienen mayor participación interna, y además realizan su trabajo no siempre en función de intereses económicos. Por otro lado, las universidades analizadas conllevan a cumplir normas para fortalecer la honestidad, solidaridad y confianza entre los trabajadores, fortaleciéndose de un código de ética, de justicia, y de ayuda mutua. Asimismo, se pudo concluir que a pesar de que los empleados no tengan sentido pertenencia, éstos se muestran comprometidos con la responsabilidad social que les corresponde, buscando así lograr la efectividad de las instituciones en cuanto a su aplicación, mostrándose voluntarios para participar en las actividades, por lo que estos recintos universitarios analizados deben crear mecanismos que permiten la participación activa de sus empleados dentro en los planes de responsabilidad social tanto interna, como externa. Esto implica que a pesar de reconocer lo significativo de la responsabilidad social como estrategia competitiva de las universidades, no han avanzado en su implementación. No cabe duda que es importante pensar en las necesidades de la comunidad, pero también lo es tomar en cuenta los intereses de quienes pueden ser considerados como principales stakeholders de la empresa: los trabajadores, grupos familiares, clientes, proveedores. Por lo que es preciso señalar, que para toda organización su prioridad pudiera ser el promover una gestión sostenible, mejorando la conservación y utilización sostenible de los recursos naturales, para coadyuvar a incrementar la calidad de vida de todos los actores involucrados. De manera tal, que al integrarse la gestión ambiental a los objetivos económicos persigan una utilización eficiente de los recursos, así como la disminución de los costos, que le permita incrementar rentabilidad y competitividad, identificando las oportunidades económicas, técnicas organizativas para prevenir o reducir la contaminación y sus riesgos. Es por esas razones, que el investigador considera que la gestión ambiental implica la utilización de mecanismos, acciones e instrumentos proporcionando garantías de control y administración racional del capital natural, a través de reglamentos, ordenanzas, decretos, normas, disposiciones institucionales, jurídicas, con el objetivo de mejorar y conservar el medio ambiente, en correspondencia con factores internos y externos, culturales, sociales, políticos, intervinientes en el desempeño ambiental de las organizaciones dentro de un marco sostenible. En términos generales, se pudo finalizar indicando la importancia de la responsabilidad social enmarcada en los elementos humanos y ambientales, regida además por una serie de regulaciones legales que garanticen su práctica dando respuestas con políticas de salud, trabajo, ética, valores morales, fundamentos de la institución, planes activistas que se basen en el bienestar social y en el desarrollo sustentable del contexto. El fin es emplearlas al máximo, constituyendo un engranaje que facilite el desarrollo sustentable, generando productividad para las Universidades públicas binacionales y el bienestar para la sociedad donde se desenvuelven.

\section{REFERENCIAS:}

García, P. (2006). Acción Empresarial Organizacional. Editorial McGraw Hill. México.

Guerra, A (2007). De la Responsabilidad Social Empresarial, a la Ética en el Cambio Organizacional. DAC- UCLA. COMPENDIUM, Número 18. Julio, 2007. Barquisimeto. Venezuela.

Libro verde (2010). Fomentar un Marco Europeo para la Responsabilidad Social de las Empresas. COMISIÓN DE LAS COMUNIDADES EUROPEAS. (CCE). Bruselas. 18.7.2010. 
Nieto, F. Fernández, R. (2004). Gestión ética para el bien común en las organizaciones. Revista Sinergia. Primera Revista Académica sobre Responsabilidad Social. Categoría: Artículo académico. Venezuela

Torres (2014). Planes de beneficios sociales de la responsabilidad social externa en las instituciones privadas de educación superior del estado Zulia. Artículo arbitrado en la Revista Arbitrada de Estudios Interdisciplinarios del Centro de Investigación de Ciencias Administrativas y Gerenciales.

Vallaeys, F. (2007). Breve marco teórico de Responsabilidad Social Universitaria. Universidad de las Américas de Puebla, Banco Interamericano de Desarrollo, http://www.udlap.mx/ SocialUniv.pdf, 01 de Mayo, 2008, México. 